\title{
Deprescription: a global need to rationalize drug prescribing
}

\author{
Subhash Vishal*, Biswadeep Das, Shailendra Handu
}

Department of Pharmacology, All India Institute of Medical Sciences, Rishikesh, Uttarakhand, India

Received: 17 October 2020

Accepted: 07 November 2020

*Correspondence:

Dr. Subhash Vishal,

Email: dr.aashu.garg@gmail.com

Copyright: (C) the author(s), publisher and licensee Medip Academy. This is an open-access article distributed under the terms of the Creative Commons Attribution Non-Commercial License, which permits unrestricted non-commercial use, distribution, and reproduction in any medium, provided the original work is properly cited.

\begin{abstract}
Appropriate prescribing and deprescription of unwanted medicines are a global concern. Polypharmacy is common in old age due to multiple comorbidities. This poses many risks that can be prevented by deprescription as a measure of planned reduction in number of medicines no longer needed. For articles to be included in this narrative review, a non-systematic search of deprescription and related term was conducted at PubMed and Google Scholar database. Articles detailing deprescription in general were included whereas those about deprescription in a particular disease or of particular drug groups were excluded. The review discusses about related terms, process of deprescription, when it is to be planned, which patients need deprescription, tools available for appropriate prescription, importance of patient oriented deprescription, actual steps involved in deprescription, present scenario, future scope of trials and formulation of guidelines for deprescription, and finally current state of deprescription in India and actions needed.
\end{abstract}

Keywords: Deprescription, Medicine withdrawal, Polypharmacy, Inappropriate medication, Older patients, Narrative review

\section{INTRODUCTION}

Health care teams, endeavour to provide right medicines to patients, with stress on prescribing 'only' right medicines to patients. This effort is recorded in history from the day of Hippocrates.

When we talk about rational prescribing, in itself, it incorporates many dimensions. Polypharmacy, adverse effects, drug interactions, potentially inappropriate medications (PIM), pharmaco-economics, utilisation of health resources, prescribing in elderly - all these converge towards a single objective of reducing unwanted medicines, termed as "deprescription".

Most of the published literature talks about deprescription in older patients. This is understandable, as with advancing age, multiple health problems necessitate prescribing and consuming more medications, resulting in chances of over-prescribing and over-consumption. Understandably further, all evidences generated for PIM are in older people, very few for PIM in the younger. But possibilities of inappropriate medicines in young cannot be denied. There are ample evidence reporting polypharmacy in young. ${ }^{1}$

In this review, we attempt to emphasize various the various aspects of deprescribing, its importance, and how and what we can contribute.

\section{SEARCH PLAN}

A non-systematic search of research database including PubMed and Google Scholar was conducted on $24^{\text {th }}$ August 2020 for articles including search term deprescription. From PubMed search, results from first 10 pages were included. Google Scholar search was done 
using sorting method both ways - by relevance and by date, and results from first 10 pages were included.

Articles common from different searches were scrutinised and inclusions were rectified accordingly. As the study progressed, further search of related terms (including PIM, polypharmacy, medication related problems, prescribing cascade, frailty, polypharmacy and young, deprescription and India) was also conducted in a similar manner to support the study. All searches were further scrutinised and sorted as per requirements. We also included previous reviews in our study.

We excluded articles related to deprescription of a particular group of drugs or disorders, focusing on deprescription as a general term.

Articles with full text available and those with restricted access but available full text via institutional portal were included. Articles with restricted access but containing required information in abstract were also included. Search results with reviews were preferred over single study for better level of evidence. Articles from references in included studies were also explored and included as per availability.

\section{SEARCH RESULTS}

First 10 pages of PubMed search showed 100 studies (10 results per page) and Google Scholar search identified 200 studies (according to relevance and date both). After merging studies from different searches for deprescription, 194 studies were sorted. 109 studies focusing on a particular disease or drugs group were excluded. Remaining 85 studies were finally included.

Similarly, search of other related terms (as quoted above) and after exploring studies referenced in above studies finally contributed to another 9 relevant studies.

Searching ("deprescription" and India) resulted in 48 results on Google Scholar, of which only 3 were found relevant. Searching same term on PubMed resulted in only 4 results with only one relevant and common with Google Scholar search. These 3 studies were also included.

\section{DEFINING RELATED TERMS}

\section{Multimorbidity}

Co-existence of more than one chronic medical health conditions is multimorbidity. With advancing age, multimorbidity becomes much commoner.

\section{Polypharmacy}

Use of multiple medications in a single patient is termed as polypharmacy. Defining polypharmacy in numerical value varies considerably between countries and regions, most common being five or more medicines daily.

Also, defining polypharmacy in terms of numbers only may neither be sufficient nor appropriate. Other factors may also play a role, like medicines prescribed that are not clinically indicated, or healthcare setting (greater number of medicines may be considered appropriate for in-patient or critical care settings). ${ }^{2}$

\section{Older people}

Health problems arise and increase with advancing age. Globally accepted age considered as old is 65 years and beyond.

\section{Frailty}

The term "frail" has a variety of clinically relevant meanings including weak, tenuous, thin, and slight; lacking normal strength; prone to be easily broken; unusually susceptible to disease or other infirmity; likely to fail or die quickly. With aging, it is a cumulative decline of different physiological systems, leading to a state of diminished reserve and increased vulnerability to various stressors. $^{3}$

\section{PIM}

Term used by Beers in 1997, can be defined as use of medications whose risks outweigh their benefits. ${ }^{4,5}$ It includes over/under-prescriptions and mis-prescriptions. ${ }^{6}$ Medication once prescribed judiciously but now no longer needed is also considered as inappropriate.

In elderly, PIMs may often be associated with increased adverse drug reactions, increased direct treatment costs, reduced drug compliance and thus morbidity reflecting in more hospitalisations and/or health expenses.

O'Mahony and co-researchers pointed out that new STOPP and START tools are better than Beers' criteria for detection of PIM. ${ }^{6}$

\section{Medication related problems}

This broader term may include drug interactions, adverse drug reactions, therapeutic failure, taking inappropriate dose or failure to take medicines (non-compliance due to any reason including number, size, shape, frequency, odd-timings, formulation of drugs, chronic use, ineffective or costly drugs).

\section{Prescribing cascade}

Prescribing cascade is use of one medication to treat the adverse effects of another medication when an ADR is misdiagnosed as a new medical condition and a new drug is added to treat it. Patient trapped in prescription cascade is exposed to encounter serious adverse health 
consequences. It must be seen differently from any drug prescribed prophylactically to prevent anticipated sideeffect of a drug (e.g. PPI given along with gastric irritants).

\section{Deprescribing}

In a systematic review, Emily Reeve and her coresearchers worked out the definition of deprescription as the process of intentional withdrawal or dose reduction of an inappropriate medication to manage polypharmacy and to improve treatment outcomes. ${ }^{7,8}$

\section{WHEN TO PLAN DEPRESCRIPTION?}

Guidelines based on evidences help clinicians to prescribe correctly indicated medicines for most of the medical conditions. But the consensus is lacking on a system for stopping medicines when no longer needed or when they may cause harm more than benefit. ${ }^{9}$

Best time to plan any deprescription is at the time of prescribing itself. When a definite indication and planned duration of prescribing a drug is not mentioned, it becomes extremely difficult to deprescribe it. If the turnover rate of in-patients/out-patients is more, it becomes harder for a prescriber to recall what the actual thought was when the drug was prescribed days or weeks before. If the quality and rationality of prescription itself is improved, and so there are minimal PIM or polypharmacy, need of deprescription will itself be minimised. For appropriateness of prescriptions, there are many tools which can aid prescribers to prescribe rationally and minimise the number of medicines. These tools can be grouped into implicit and explicit ones. Implicit tools contain questions to be asked by a prescriber to identify whether a drug is still required, whereas explicit tools list medications to be reviewed by name. ${ }^{9}$ Implicit tools are drug or disease oriented, easy to apply, with little or no clinical judgment. However, explicit tools might not take into account all factors (including comorbid conditions) that define high quality health care for the patient. ${ }^{5}$

Tools to improve prescribing include: ${ }^{10}$ Beers' criteria, Canadian criteria or improved prescribing in elderly tool, STOPP/START, medication appropriateness index (MAI), fit for the aged criteria (FORTA), the assess, review, minimize, optimise, reassess (ARMOR) tool, confirm, estimate, assess, sort, estimate (CEASE), good palliative-geriatric practice (GPGP) algorithm, geriatric risk assessment Medguide (GRAM), prescribing optimization method (POM), anticholinergic risk scale, drug burden index, and PRISCUS list.

\section{WHO NEEDS DEPRESCRIBING?}

Deprescription is needed to be considered in each and every prescription in which medicines are prescribed for a duration longer than an acute course, whether in young or in old patients. However, it is obligatory in older patients due to multiple comorbidities and factors associated with different pattern of prescribing: reduced physiological reserve; frailty; multimorbidity leading to polypharmacy, which in turn leads to more adverse drug reactions and interactions, that further leads to less compliance; and limited evidence/research in older people. $^{11}$

Appropriateness of prescription, and so deprescription, of antibiotics may also be seen in the light of existing local antibiotic policy and antimicrobial stewardship programme.

\section{PATIENT AWARENESS}

Inputs for deprescription are needed from both prescriber as well as patient. Patients, especially older ones, should know about the option of deprescription. General thinking among older patients is that to be in optimal state of health, they need to consume all medicines prescribed by their clinician.

Additionally, in India, patients also take alternative therapies. Apart from modern medicine, AYUSH (Ayurveda, Yoga, Unani, Siddha and Homoeopathy) system of medicine is also common, more often among older patients. When patients are enquired about the medicines, they are taking at any point of time, they may not report these drugs considering them as natural/herbal products without any adverse effects. In such cases, it becomes difficult to assess the actual extent of polypharmacy, and many a times, the culprit drug for any adverse drug reaction or interaction.

\section{PATIENT ORIENTED DEPRESCRIPTION AND SHARED DECISION MAKING}

A well-informed patient can actively participate in shared decision making and correct selection of $\operatorname{drug}(\mathrm{s})$ to be deprescribed becomes easier and effective. Contrary to common belief, older patients actively participate in the process of deprescription and do not take deprescription as withdrawal of care. ${ }^{12}$

Active involvement of patient in deprescription process can give more accurate clue about problem drug(s), drugs causing adverse reactions, drugs with non-compliance, drugs taken irregularly/SOS by patients, self-discontinued $\operatorname{drug}(\mathrm{s})$ or $\operatorname{drug}(\mathrm{s})$ no longer needed, and so chances of getting a positive outcome are much increased.

\section{BARRIERS TO AND ENABLERS OF DEPRESCRIPTION ${ }^{11}$}

\section{Barriers}

Limited consultation/discussion time; prior unfruitful experiences of withdrawing medications; lack of patient interest/involvement in deprescription process; fear of 
return of original medical condition and/or medication withdrawal effect; fear of any harm or even death; disagreement between patient and prescriber for usefulness/appropriateness of the drug in question

\section{Enablers}

Patient will to deprescribe/shorten regimen; active patient involvement with good communication, including discussion of detailed disease and drug history; sufficient options offered to choose regimen; detailed information regarding possible outcomes both positive as well as negative; encouragement and assurance of care from prescriber, explaining benefits of deprescription

\section{STEPS FOR DEPRESCRIPTION}

\section{Collect drug information}

Thorough discussion with active patient participation for collecting good drug/disease course history is key to identify the target $\operatorname{drug}(s)$ that can be planned for deprescription. What patient thinks about usefulness/ uselessness of drugs in regimen. Is there any drug that patient self-deprescribed already? Reasons for not taking that particular drug? Is/are there any drug(s) that patient taking apart from current prescriber- from another clinician for different sets of problems or from a practitioner of alternative medicine?

\section{Identify drug that can be planned for deprescription}

Drugs indicated for limited duration/specific purpose, that served the purpose of prescription and no longer needed; clinical condition changed/ceased for which it was prescribed; drugs that are no longer appropriate i.e. PIM (appropriateness can be evaluated with help of tools discussed above), drugs causing adverse reaction(s); $\operatorname{drug}(\mathrm{s})$ not serving the purpose of prescription- may be ineffective; patient non-compliant for that particular drug due to any reason,

\section{Workup a planned deprescription regimen}

Goals to be achieved from deprescription must be clearly defined; expected outcomes; drugs under consideration for deprescription will be tapered or weaned?; quantify possible emergence of original medical condition; identify possibilities of masking/unmasking of adverse reactions of other drugs in regimen, if any; plan deprescription of inappropriate medicines for goodness of patient, even if lack of interest from patient, especially older one for involvement in process; follow available literature, though limited, if available for deprescription of that particular drug and; ethical and legal aspects should also be honoured and addressed in planning to avoid any future possible consequences.

\section{Discuss plan with patient}

Assure deprescription is not withdrawal of care or cessation of treatment; discuss with patient about plan, proposed regimen, possible outcomes and alternatives/ options in details; never commit a positive outcome, rather informing any possible risk/outcome in advance will add up to the efficacy of deprescription process; document each and every step including points discussed with patient to avoid any legal complication.

\section{Frequent follow-up and prompt support}

Patient compliance with deprescription is better if there are clear benefits in terms of patient interests, any adverse outcome including adverse drug withdrawal effect to be addressed, patient's will must be respected if original medical condition reappears and patient is willing to restart deprescribed drug. It must not be seen as failure of clinician or failure of deprescription process; drugs to be tapered gradually need more frequent and careful followup; half-life period of drugs in question may give clue how frequent will follow-up will be needed; after a long duration, there may be a need of restarting the drug and regular follow-up is required to identify that very timepoint.

\section{DEPRESCRIPTION TRIALS AND GUIDELINE}

Polypharmacy and drug related problems are more common in older people. Even when evidence is lacking for prescription in older populations, evidence for deprescription are further scanty. Encouraging part is that awareness is increasing and trials are in progress. Need of guidelines for deprescription is now being felt globally and efforts are being poured in form of research. Amy Theresa page and others reviewed 116 RCTs investigating deprescription of single medications and discussed 31 studies in detail. ${ }^{11}$ Barbara Farrell and coworkers reviewed methodology for developing deprescribing guidelines. ${ }^{13}$

\section{DEPRESCRIPTION IN INDIA}

Studies and efforts in the direction of discontinuing/ decreasing inappropriate medicines are going since long. But specific steps in direction of deprescription as an effective tool are still lacking. Concept of deprescription in India is new for majority of health care professionals. In India, health infrastructure largely rests on the shoulders of primary health care, where medical graduates render medical services to the patients. Awareness is emerging but present knowledge of a medical graduate regarding deprescription cannot be accurately cited.

\section{CONCLUSION}

Use of inappropriate drugs is a rising global problem. Periodic review of medicines taken by patient is required for optimisation of therapy. Deprescription is planned reduction/stopping of an unwanted/inappropriate medicine. It is very much personalised and an ongoing process. Patient engagement in process is crucial for 
successful deprescription. Risks and benefits must be weighed and discussed with patient. Several tools exist to facilitate prescription but there is lack of sufficient guidelines and tools for deprescription. Trials are needed to generate evidence for framing guidelines for deprescription and concept needed to be incorporated in curriculum to impart knowledge of deprescription at undergraduate level of medical education.

Funding: No funding sources

Conflict of interest: None declared

Ethical approval: Not required

\section{REFERENCES}

1. Menditto E, Miguel GA, Juste MA, Plou PB, Aza Pascual-Salcedo $M$ et al. Patterns of multimorbidity and polypharmacy in young and adult population: Systematic associations among chronic diseases and drugs using factor analysis. PloS One. 2019;14(2):e0210701.

2. Masnoon N, Shakib S, Kalisch-Ellett L, Caughey GE. What is polypharmacy? A systematic review of definitions. BMC Geriatr. 2017;17(1):230.

3. Walston J, Hadley EC, Ferrucci L, Guralnik JM, Newman AB, Studenski SA et al. Research agenda for frailty in older adults: toward a better understanding of physiology and etiology: summary from the American Geriatrics Society/National Institute on Aging Research Conference on Frailty in Older Adults. J Am Geriatr Soc. 2006;54(6):9911001.

4. Beers MH. Explicit Criteria for Determining Potentially Inappropriate Medication Use by the Elderly: An Update. Arch Intern Med. 1997;157(14):1531-6.

5. Spinewine A, Schmader KE, Barber N, Hughes C, Lapane KL, Swine C, et al. Appropriate prescribing in elderly people: how well can it be measured and optimised? Lancet. 2007;370(9582):173-84.

6. O'Connor MN, Gallagher P, O'Mahony D. Inappropriate prescribing: criteria, detection and prevention. Drugs Aging. 2012;29(6):437-52.

7. Michael WC. Deprescribing: Achieving Better Health Outcomes for Older People through Reducing Medications. J Pharm Pract Res. 2003;33.

8. Reeve E, Gnjidic D, Long J, Hilmer S. A systematic review of the emerging definition of 'deprescribing' with network analysis: implications for future research and clinical practice. Br J Clin Pharmacol. 2015;80:1254-68.

9. Le Bosquet K, Barnett N, Minshull J. Deprescribing: Practical Ways to Support Person-Centred, Evidence-Based Deprescribing. Pharmacy (Basel). 2019;7(3):129.

10. Gokula M, Holmes HM. Tools to reduce polypharmacy. Clin Geriatr Med. 2012;28(2):323-41.

11. Page AT, Potter K, Clifford R, Etherton-Beer C. Deprescribing in older people. Maturitas. 2016;91:115-34.

12. Schuling J, Gebben H, Veehof LJ, Haaijer-Ruskamp FM. Deprescribing medication in very elderly patients with multimorbidity: the view of Dutch GPs. A qualitative study. BMC Fam Pract. 2012;13:56.PMC3391990.

13. Farrell B, Pottie K, Rojas-Fernandez CH, Bjerre LM, Thompson W, Welch V. Methodology for Developing Deprescribing Guidelines: Using Evidence and GRADE to Guide Recommendations for Deprescribing. PLoS One. 2016;11(8):e0161248.

Cite this article as: Vishal S, Das B, Handu S. Deprescription: a global need to rationalize drug prescribing. Int J Basic Clin Pharmacol 2020;9:191721. 